\title{
Identification of key pathogenic genes of sepsis based on the Gene Expression Omnibus database
}

\author{
XINXING LU* , LU XUE*, WENBIN SUN, JILU YE, ZHIYUN ZHU and HAIFENG MEI \\ Department of Intensive Medicine, Taizhou People's Hospital, Taizhou, Jiangsu 225300, P.R. China
}

Received May 2, 2017; Accepted November 10, 2017

DOI: $10.3892 / \mathrm{mmr} .2017 .8258$

\begin{abstract}
Sepsis is a life-threatening condition in which an uncontrolled inflammatory host response is triggered. The exact pathogenesis of sepsis remains unclear. The aim of the present study was to identify key genes that may aid in the diagnosis and treatment of sepsis. mRNA expression data from blood samples taken from patients with sepsis and healthy individuals was downloaded from the Gene Expression Omnibus database and differentially expressed genes (DEGs) between the two groups were identified. Gene Ontology and Kyoto Encyclopedia of Genes and Genomes (KEGG) pathway analysis, and protein-protein interaction (PPI) network construction, was performed to investigate the function of the identified DEGs. Furthermore, for validation of these results, the expression levels of several DEGs were analyzed by reverse transcription quantitative-polymerase chain reaction (RT-qPCR) in three patients with sepsis and three healthy blood samples to support the results obtained from the bioinformatics analysis. Receiver operating characteristic analyses were also used to analyze the diagnostic ability of the identified DEGs for sepsis. The results demonstrated
\end{abstract}

Correspondence to: Dr Xinxing Lu, Department of Intensive Medicine, Taizhou People's Hospital, 210 Yingchun East Road, Hailing, Taizhou, Jiangsu 225300, P.R. China

E-mail: luxinxingjs@163.com

"Contributed equally

Abbreviations: ADM, adrenomedullin; AGTRAP, angiotensin II receptor-associated protein; ALOX5, arachidonate 5-lipoxygenase; DEGs, differentially expressed genes; ENTPD1, ectonucleoside triphosphate diphosphohydrolase 1; FDR, false discovery rate; GEO, Gene Expression Omnibus; GO, Gene Ontology; IRAK3, interleukin 1 receptor-associated kinase 3; KEGG, Kyoto Encyclopedia of Genes Genomes; MMP9, matrix metallopeptidase 9; PPI, protein-protein interaction; RT-qPCR, reverse transcription quantitative-polymerase chain reaction; S100A8, S100 calcium-binding protein A8; S100A9, S100 calcium-binding protein A9; SAE, sepsis-associated encephalopathy; TLR, toll-like receptor

Key words: sepsis, gene expression, protein-protein interaction network, biomarkers that a total of 4,402 DEGs, including 1,960 upregulated and 2,442 downregulated genes, were identified between patients with sepsis and healthy individuals. KEGG pathway analysis revealed that 39 DEGs were significantly enriched in toll-like receptor signaling pathways. The top 20 upregulated and downregulated DEGs were used to construct the PPI network. Hub genes with high degrees, including interleukin 1 receptor-associated kinase 3 (IRAK3), S100 calcium-binding protein (S100)A8, angiotensin II receptor-associated protein (AGTRAP) and S100A9, were demonstrated to be associated sepsis. Furthermore, RT-qPCR results demonstrated that IRAK3, adrenomedullin (ADM), arachidonate 5-lipoxygenase (ALOX5), matrix metallopeptidase 9 (MMP9) and S100A8 were significantly upregulated, while ectonucleoside triphosphate diphosphohydrolase 1 (ENTPD1) was upregulated but not significantly, in blood samples from patients with sepsis compared with healthy individuals, which was consistent with bioinformatics analysis results. Therefore, AGTRAP, IRAK3, ADM, ALOX5, MMP9, S100A8 and ENTPD1 were identified to have potential diagnostic value in sepsis. In conclusion, dysregulated levels of the AGTRAP, IRAK3, ADM, ALOX5, MMP9, S100A8 and ENTPD1 genes may be involved in sepsis pathophysiology and may be utilized as potential diagnostic biomarkers or therapeutic targets.

\section{Introduction}

Sepsis is a life-threatening condition that is caused by the entry of bacteria, fungi, viruses or parasites to the bloodstream, which subsequently triggers a systemic uncontrolled inflammatory response (1). Sepsis is be divided into three classifications with increasing severity: Sepsis, severe sepsis and septic shock (2). Severe sepsis is often complicated by septic shock and multiple organ dysfunction syndrome, and the incidence and mortality rate of sepsis is higher in elderly patients $(3,4)$. Additionally, sepsis syndrome and septic shock are the major cause of mortality in intensive care units. More than 750,000 cases of sepsis occur in the United States each year (5), which result in more than 210,000 deaths per year (6).

At present, the exact mechanism of sepsis pathophysiology is not clear. A previous study reported that the pathophysiology includes highly complex interactions between invading microorganisms, the innate and adaptive immune systems of the host and multiple downstream events, which lead to organ 
dysfunction (7). In addition, Calvano et al (8) demonstrated that $\sim 25 \%$ of normal human gene expression was altered in sepsis. Hypermorphic genetic variation in TLR1 is reported to also have roles in the clinical outcomes of sepsis (9). Despite clinical advances, the mortality rate of sepsis has not considerably reduced in the last 10 years. (10). Early diagnosis and treatment is crucial in order to reduce the mortality rate in patients with sepsis.

The present study aimed to identify differentially expressed genes (DEGs) in sepsis using integrated analysis. Functional enrichment analysis using the Gene Ontology (GO) and Kyoto Encyclopedia of Genes and Genomes (KEGG) was subsequently performed to investigate the biological functions of the identified DEGs. A protein-protein interaction (PPI) network was subsequently constructed from the top 40 DEGs (20 upregulated and 20 downregulated). Furthermore, reverse transcription quantitative-polymerase chain reaction (RT-qPCR) was performed to analyze the expression of candidate DEGs in patients with sepsis and healthy individuals to validate bioinformatics results. Receiver operating characteristic (ROC) analysis was performed to analyze the diagnostic ability of the identified DEGs in sepsis. The DEGs in the present study may have potential as diagnostic biomarkers or aid the development of novel therapeutics.

\section{Materials and methods}

Gene expression profiles. In the current study, relevant datasets from the Gene Expression Omnibus (GEO) database (http://www.ncbi.nlm.nih.gov/geo/) with the keywords 'sepsis' [Medical Subject Headings (MeSH) Terms] OR 'sepsis' (All Fields) AND 'Homo sapiens' [Organism name (porgn)] AND 'gse' (Filter). The study type was characterized as 'expression profiling by array.' All selected datasets consisted of genome-wide expression data from blood samples of patients with sepsis and/or healthy samples. Only standardized or primary datasets were included in this study. Two datasets were ultimately selected for screening. The details of datasets GSE69528 (11) and GSE46955 (12) are presented in Table I.

Identification of DEGs. The raw expression data of patients with sepsis was obtained from the GEO database. The significantly DEGs were identified by comparison between patients with sepsis and healthy controls. P-values were combined using the meta-analysis for microarrays (metaMA version 3.3.3) package (13) in the R software 3.3.3 (https://www.r-project.org/). The false discovery rate (FDR) was calculated for multiple testing corrections of the raw P-value through the Benjamin and Hochberg method $(14,15)$. The threshold for DEG identification was set as FDR $<0.05$.

Functional and pathway enrichment analyses of DEGs. To obtain the biological function and signaling pathways of DEGs, GeneCodis3 (http://genecodis.cnb.csic.es/analysis) was used for the GO (http://www.geneontology.org/) annotation and KEGG (http://www.genome.jp/kegg/pathway.html) pathway enrichment of DEGs. The threshold for GO and KEGG analysis of DEGs was set at FDR $<0.05$.
PPI network construction. To gain insight into the interaction between DEGs and proteins, the BioGRID database (http://thebiogrid.org) was used to obtain the predicted interactions between the top 40 proteins encoded by DEGs (20 upregulated and 20 downregulated) and other associated proteins. The PPI network was subsequently visualized using Cytoscape software 3.3.0 (http://cytoscape.org/). A node in the PPI network denotes a protein and an edge denotes interactions between proteins.

$R T$-qPCR validation. Blood samples were obtained from 3 male patients (average, 71) diagnosed with sepsis and 3 male healthy individuals (average, 71) from Taizhou People's Hospital during November, 2016. The collected blood was then immediately frozen in liquid nitrogen. All participating individuals enrolled in the present study provided informed consent and approval was obtained from the ethics committee of Taizhou People's Hospital (Taizhou, China). Total RNA was extracted from the sepsis and control blood samples using TRIzol ${ }^{\text {TM }}$ reagent (Invitrogen; Thermo Fisher Scientific, Inc., Waltham, MA, USA) according to the manufacturer's protocol. cDNA was synthesized using the SuperScript III First-Strand Synthesis System (Invitrogen; Thermo Fisher Scientific, Inc.) for $5 \mathrm{~min}$ at $65^{\circ} \mathrm{C}$ followed by $60 \mathrm{~min}$ at $42^{\circ} \mathrm{C}$ and $5 \mathrm{~min}$ at $72^{\circ} \mathrm{C}$. qPCR was performed using the SYBR Green PCR Master Mix (Applied Biosystems; Thermo Fisher Scientific, Inc.) with the Applied Biosystems 7500 RT-PCR system (Applied Biosystems; Thermo Fisher Scientific, Inc.). The amplification process was performed under the following conditions: $15 \mathrm{~min}$ at $95^{\circ} \mathrm{C}$ followed by 40 cycles of $10 \mathrm{sec}$ at $95^{\circ} \mathrm{C}, 30 \mathrm{sec}$ at $55^{\circ} \mathrm{C}, 32 \mathrm{sec}$ at $72^{\circ} \mathrm{C}$, and $15 \mathrm{sec}$ at $95^{\circ} \mathrm{C}$, $60 \mathrm{sec}$ at $60^{\circ} \mathrm{C}$ and $15 \mathrm{sec}$ extension at $95^{\circ} \mathrm{C}$. The sequences of reverse and forward primers for all of the genes analyzed were as follows: IRAK3 forward (F), CAGCCAGTCTGAGGTTAT GTTT and reverse (R), TTGGGAACCAACTTTCTTCACA; ADM F, CTTATTCGGCCCCAGGACATG, and R, GCGACG TTGTCCTTGTCCTTA; ALOX5 F, TGAGCCAGTTCCAGG AAAACG and R, ATGGCCACACTGTTCGGAATC; MMP9 F, TGTACCGCTATGGTTACACTCG and R, GGCAGGGAC AGTTGCTTCT; S100A8 F, CAGCCCTGCATGTCTCTT GTC and R, CCCTGTAGACGGCATGGAAAT; SOCS3 F, CATGGAGAGGGACCCAGCATA and R, GACATTCCC AGTGCTCAGCTG; CD14 F, GACCTAAAGATAACCGGC ACC and R, GCAATGCTCAGTACCTTGAGG; ENTPD1 F, AGGTGCCTATGGCTGGATTAC and R, CCAAAGCTC CAAAGGTTTCCT; GAPDH F, GGAGCGAGATCCCTC CAAAAT and R, GGCTGTTGTCATACTTCTCATGG.

GAPDH was used as an internal control for gene detection. The experiment was repeated three times. The relative expression of genes was calculated using the $2^{-\Delta \Delta c q}$ equation (16). Results are presented as fold change (2- $2^{-\Delta \Delta \mathrm{cq}}$; patient/control) values; $2^{-\Delta \Delta \mathrm{cq}}>1$ indicates an upregulated gene and $2^{-\Delta \Delta \mathrm{cq}}<1$ indicates a downregulated gene.

ROC analysis. ROC analysis was performed to assess the diagnostic value of differentially expressed microRNA targets, using the pROC package 3.3.3 (http://web.expasy.org/pROC/) in the R software 3.3 .3 (https://www.r-project.org/). An ROC curve was generated and a binomial exact confidence interval was calculated for the area under the curve (AUC). 
Table I. mRNA expression datasets of sepsis employed in the current study.

\begin{tabular}{|c|c|c|c|c|c|}
\hline GEO accession no. & Author & Year & Platform & Samples (N:P) & (Refs.) \\
\hline GSE69528 & Pankla et al & 2015 & $\begin{array}{l}\text { GPL10558 Illumina HumanHT-12 V4.0 } \\
\text { Expression BeadChip }\end{array}$ & $28: 29$ & (11) \\
\hline GSE46955 & Wu et al & 2014 & $\begin{array}{l}\text { GPL6104 Illumina HumanRef-8 v2.0 } \\
\text { Expression BeadChip }\end{array}$ & $12: 16$ & (12) \\
\hline
\end{tabular}

GEO, Gene Expression Omnibus; N, normal individual; P, patients with sepsis.

Table II. Top 20 differentially expressed genes between sepsis and control samples within GSE69528 and GSE46955 datasets.

\begin{tabular}{|c|c|c|c|c|c|}
\hline NCBI ID & Symbol & Combined ES & P-value & FDR & Upregulated/downregulated \\
\hline 6279 & S100A8 & 3.624648 & $<0.001$ & $<0.001$ & Upregulated \\
\hline 2207 & FCER1G & 3.597326 & $<0.001$ & $<0.001$ & Upregulated \\
\hline 199675 & C19orf59 & 3.516052 & $<0.001$ & $<0.001$ & Upregulated \\
\hline 4783 & NFIL3 & 3.510366 & $<0.001$ & $<0.001$ & Upregulated \\
\hline 55766 & $\mathrm{H} 2 \mathrm{AFJ}$ & 3.477834 & $<0.001$ & $<0.001$ & Upregulated \\
\hline 6280 & S100A9 & 3.438282 & $<0.001$ & $<0.001$ & Upregulated \\
\hline 338339 & CLEC4D & 3.394467 & $<0.001$ & $<0.001$ & Upregulated \\
\hline 5265 & SERPINA1 & 3.378025 & $<0.001$ & $<0.001$ & Upregulated \\
\hline 6283 & S100A12 & 3.329135 & $<0.001$ & $<0.001$ & Upregulated \\
\hline 54757 & FAM20A & 3.188326 & $<0.001$ & $<0.001$ & Upregulated \\
\hline 8836 & GGH & 3.15312 & $<0.001$ & $<0.001$ & Upregulated \\
\hline 2629 & GBA & 3.062176 & $<0.001$ & $<0.001$ & Upregulated \\
\hline 2319 & FLOT2 & 3.016056 & $<0.001$ & $<0.001$ & Upregulated \\
\hline 3017 & HIST1H2BD & 2.983884 & $<0.001$ & $<0.001$ & Upregulated \\
\hline 53831 & GPR84 & 2.97356 & $<0.001$ & $<0.001$ & Upregulated \\
\hline 55332 & DRAM1 & 2.92859 & $<0.001$ & $<0.001$ & Upregulated \\
\hline 8277 & TKTL1 & -2.90765 & $<0.001$ & $<0.001$ & Downregulated \\
\hline 57085 & AGTRAP & 2.902596 & $<0.001$ & $<0.001$ & Upregulated \\
\hline 11213 & IRAK3 & 2.897167 & $<0.001$ & $<0.001$ & Upregulated \\
\hline 10409 & BASP1 & 2.846037 & $<0.001$ & $<0.001$ & Upregulated \\
\hline
\end{tabular}

ES, enrichment score; FDR, false discovery rate; NCIB, national center for biotechnology information.

Statistical analysis. All statistical analyses were performed using GraphPad Prism 5 software (GraphPad Software, La Jolla, CA, USA). For the RT-qPCR validation experiment, the difference in mRNA expression between patients with sepsis and healthy controls was statistically analyzed by one-way analysis of variance. Data is presented as the mean \pm standard deviation. $\mathrm{P}<0.05$ was considered to indicate a statistically significant difference.

\section{Results}

DEG analysis. A total of 4,402 DEGs were identified with the threshold of FDR $<0.05$, including 1,960 upregulated DEGs and 2,442 downregulated DEGs in samples from patients with sepsis compared with healthy individuals. The top 20 DEGs are listed in Table II and a heatmap of the top 100 DEGs is presented in Fig. 1.
Functional and pathway enrichment analyses of DEGs. The 4,402 DEGs were analyzed by GO term and KEGG pathway enrichment. A total of 4,102 DEGs were recognized. GO and KEGG pathway analyses of the top 20 DEGs demonstrated that 'signal transduction', 'regulation of transcription, DNA-dependent' and 'apoptotic process' were the most enriched biological process (BP) terms (Fig. 2). 'Cytoplasm' and 'protein binding' were the most enriched cellular component (Fig. 3) and molecular function (Fig. 4) terms, respectively. 'Toll-like receptor signaling pathway' was among the significantly enriched KEGG pathways associated with sepsis development (Fig. 5).

PPI network construction. To identify the interactions between the DEGs, a PPI network was constructed and visualized using Cytoscape. PPI networks of the top 20 upregulated and top 20 downregulated DEGs are presented in Figs. 6 and 7, respectively. 


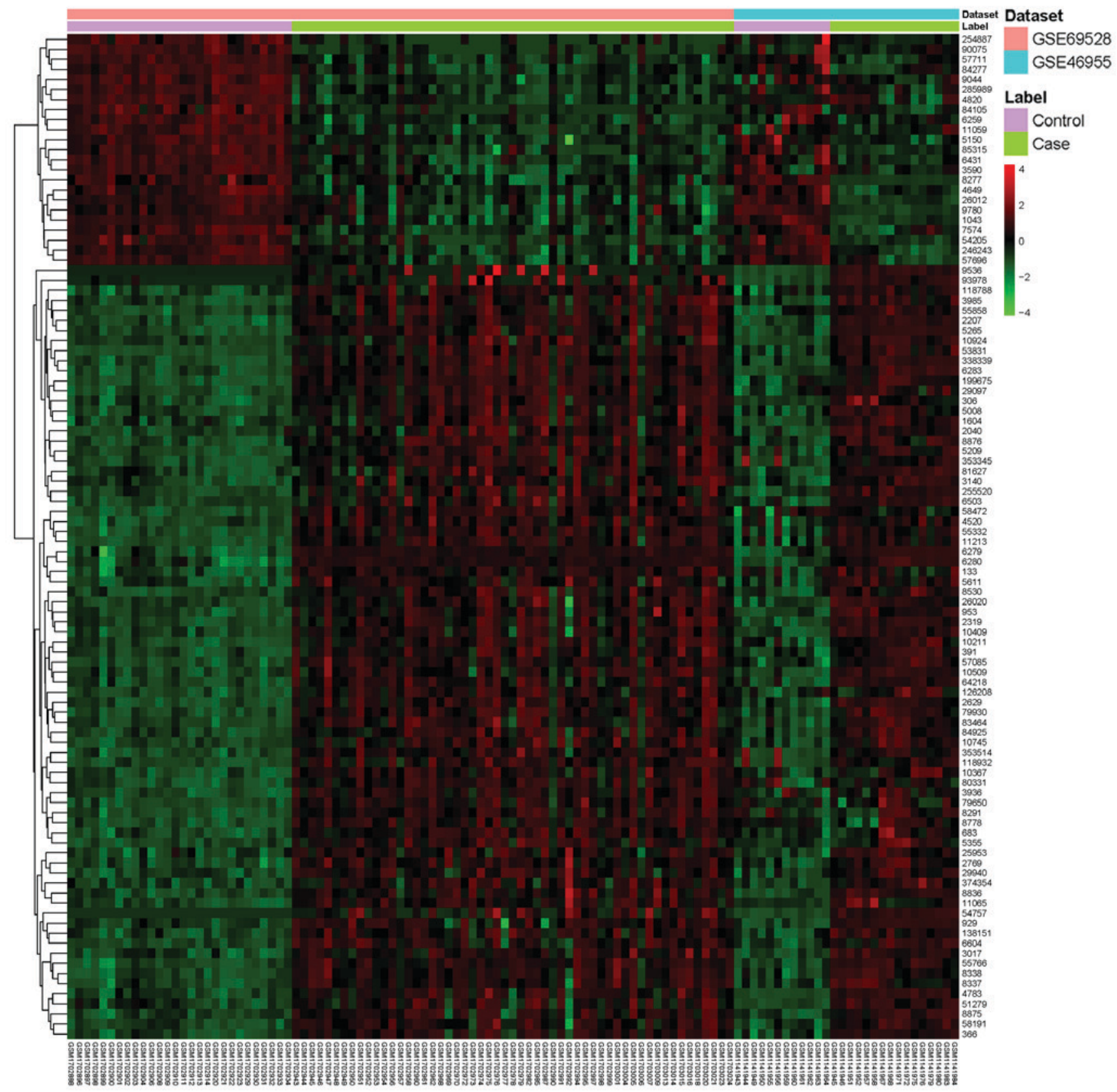

Figure 1. Heatmap of the top 100 DEGs. The diagram represents the result of a two-way hierarchical clustering of the top 100 DEGs and samples. The clustering was constructed using the complete-linkage method in combination with the Euclidean distance. Each row represents a DEG and each column represents a sample. The DEG clustering tree is presented on the left. The color scale illustrates the relative level of DEG expression: Red, lower than the reference expression; green, higher than the reference expression. DEG, differentially expressed gene.

Nodes with a high degree (proteins encoded by DEGs interact with $>5$ other proteins encoded by other genes) are described as hub proteins. As demonstrated in Fig. 6, the network for upregulated DEGs consisted of 409 nodes and 443 edges. The most important upregulated hub proteins were histone cluster $2 \mathrm{H} 2 \mathrm{~A}$ family member C (degree, 92), angiotensin II receptor-associated protein (AGTRAP; degree, 74), flotillin 2 (degree, 46), S100 calcium-binding protein (S100)A8 (degree, 43), glucosylceramidase $\beta$ (degree, 35), S100A9 (degree, 30 ), serpin family A member 1 (degree, 29), brain abundant membrane-attached signal protein 1 (degree, 20), $\gamma$-glutamyl hydrolase (degree, 18), interleukin 1 receptor-associated kinase 3 (IRAK3; degree, 18), histone cluster $1 \mathrm{H} 2 \mathrm{~B}$ family member D (degree, 13) and nuclear factor interleukin 3-regulated (degree, 11). In the downregulated DEG PPI network, there were 224 nodes and 227 edges. In Fig. 7 for the downregulated DEG network, the most important hub proteins were receptor-like tyrosine kinase (degree, 75), serine and arginine rich splicing factor 6 (degree, 45), B-TFIID TATA-box binding protein-associated factor 1 (degree, 24), cytochrome $\mathrm{C}$, somatic (degree, 18) and myosin IXA (degree, 11).

$R T$-qPCR validation. To verify the results of the bioinformatics analyses, the expression levels of selected DEGs were quantified by RT-qPCR in three sepsis and three healthy blood samples. A total of 6 DEGs, including IRAK3, adrenomedullin (ADM), 


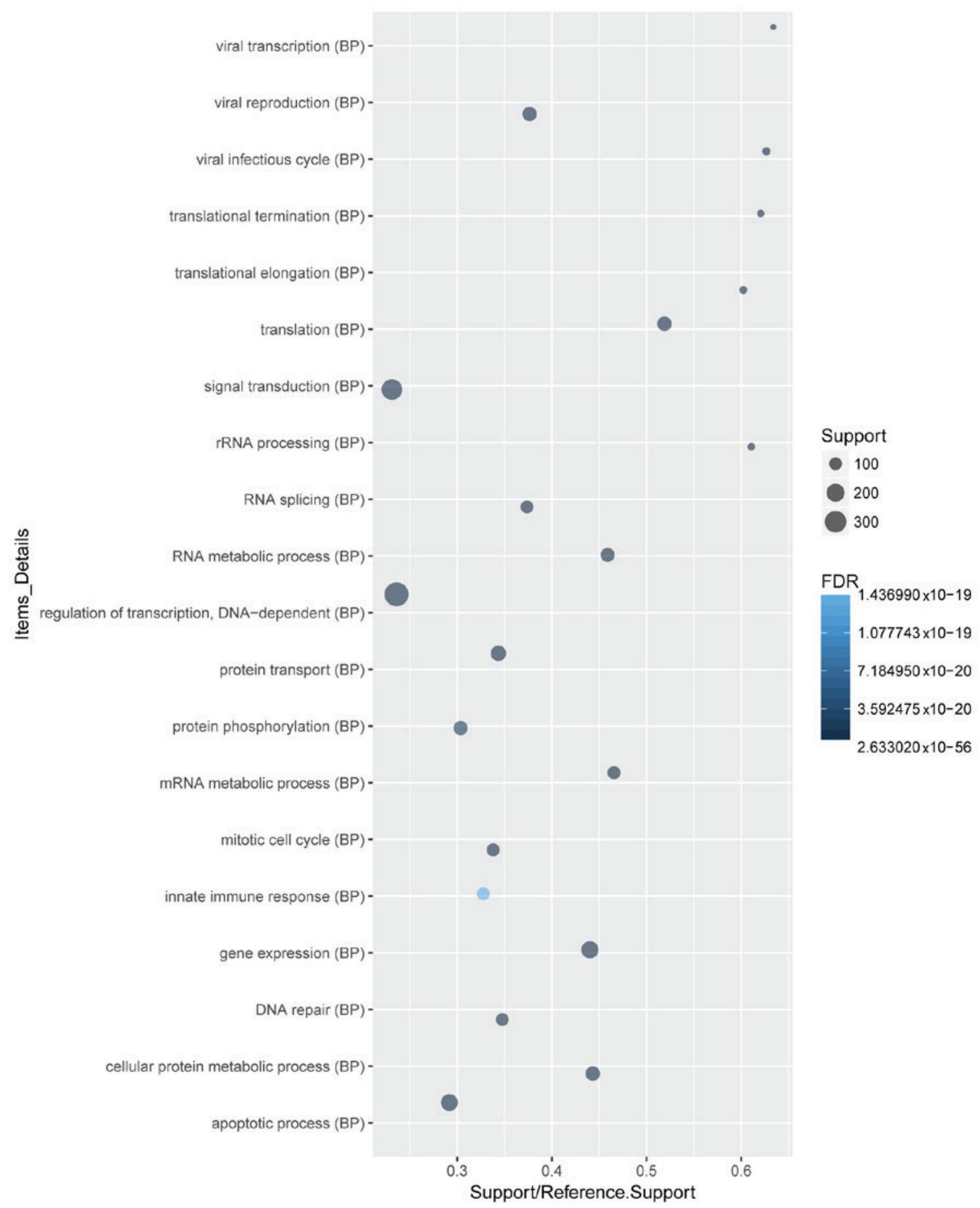

Figure 2. Top 20 significant BP terms that differentially expressed genes within GSE69528 and GSE46955 datasets were enriched in. BP, biological process; GO, gene ontology; FDR, false discovery rate.

arachidonate 5-lipoxygenase (ALOX5), matrix metallopeptidase 9 (MMP9), S100A8 and ectonucleoside triphosphate diphosphohydrolase 1 (ENTPD1) were selected as candidate genes based on the previous literature reviews $(1,17-21)$. IRAK3 $(\mathrm{P}<0.01)$, ADM $(\mathrm{P}<0.05)$, ALOX5 $(\mathrm{P}<0.001)$, MMP9 $(\mathrm{P}<0.05)$ and $\mathrm{S} 100 \mathrm{~A} 8(\mathrm{P}<0.05)$ were significantly upregulated in the blood samples of patients with sepsis compared with those of healthy controls (Fig. 8). No significant difference was identified between the ENTPD1 mRNA expression in blood samples from patients with sepsis and healthy controls in the present study (Fig. 8).
ROC curve analysis. ROC curve analyses were performed and the AUC was calculated to assess the discriminatory ability of several DEGs in the GEO dataset. The AUC of 7 DEGs, including AGTRAP (1.000), IRAK3 (1.000), ADM (0.994), ALOX5 (0.916), MMP9 (0.967), S100A8 (1.000) and ENTPD1 (0.984) was >0.9 (Fig. 9). AGTRAP, IRAK3 and S100A8 had the largest AUC among these 7 DEGs. For sepsis diagnosis, the 1-specificity (proportion of false positive results) and sensitivity (proportion of true positive results) values, respectively, were as follows: AGTRAP, 100 and 100\%; IRAK3, 100 and 100\%; ADM, 100 and 96.6\%; ALOX5, 100 and 82.8\%; 


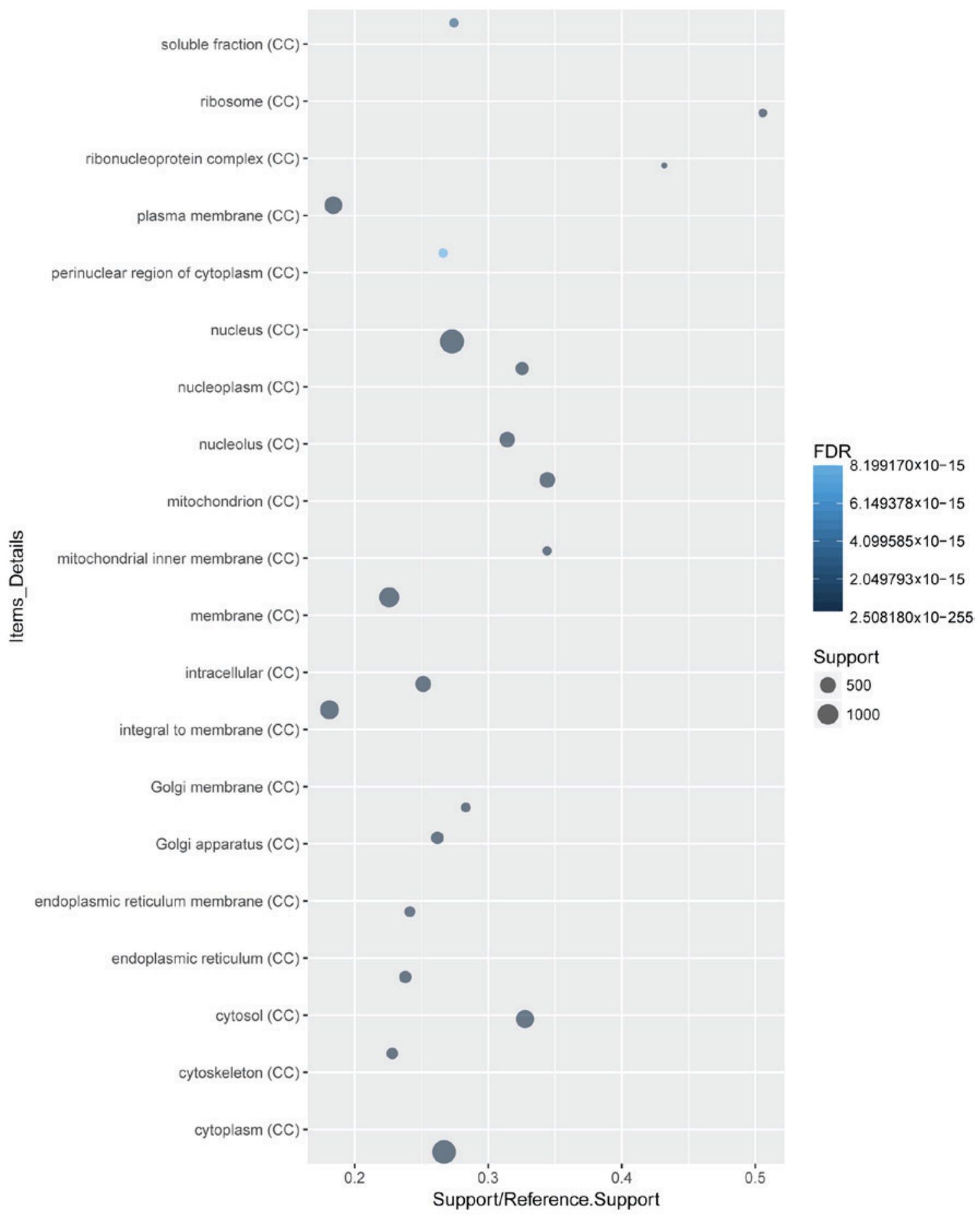

Figure 3. Top 20 significant CC terms that differentially expressed genes within GSE69528 and GSE46955 datasets were enriched in. CC, cellular component; $\mathrm{GO}$, gene ontology; FDR, false discovery rate.

MMP9, 100 and 89.7\%; S100A8, 100 and 100\%; and ENTPD1, 100 and $93.1 \%$

\section{Discussion}

Sepsis is a prevalent condition that is one of the major causes of mortality in hospitalized patients. An understanding of the mechanisms underlying sepsis pathophysiology is vital in order to develop effective, novel therapeutics and diagnostic tools. In the present study, a total of 4,402 DEGs, including 1,960 upregulated and 2,442 downregulated DEGs, were identified to be associated with sepsis. These DEGs were significantly enriched in the TLR signaling pathways. Several hub genes with high degrees, including IRAK3, S100A8, AGTRAP and S100A9, were subsequently identified in the PPI networks consisting of the top 20 upregulated and top 20 downregulated DEGs. Candidate genes IRAK3, ADM, ALOX5, MMP9, S100A8 and ENTPD1 were then validated by RT-qPCR to confirm conclusions drawn from the bioinformatics analysis. In addition, the DEGs AGTRAP, IRAK3, ADM, ALOX5, MMP9, S100A8 and ENTPD1 were also identified to have diagnostic value in sepsis. 


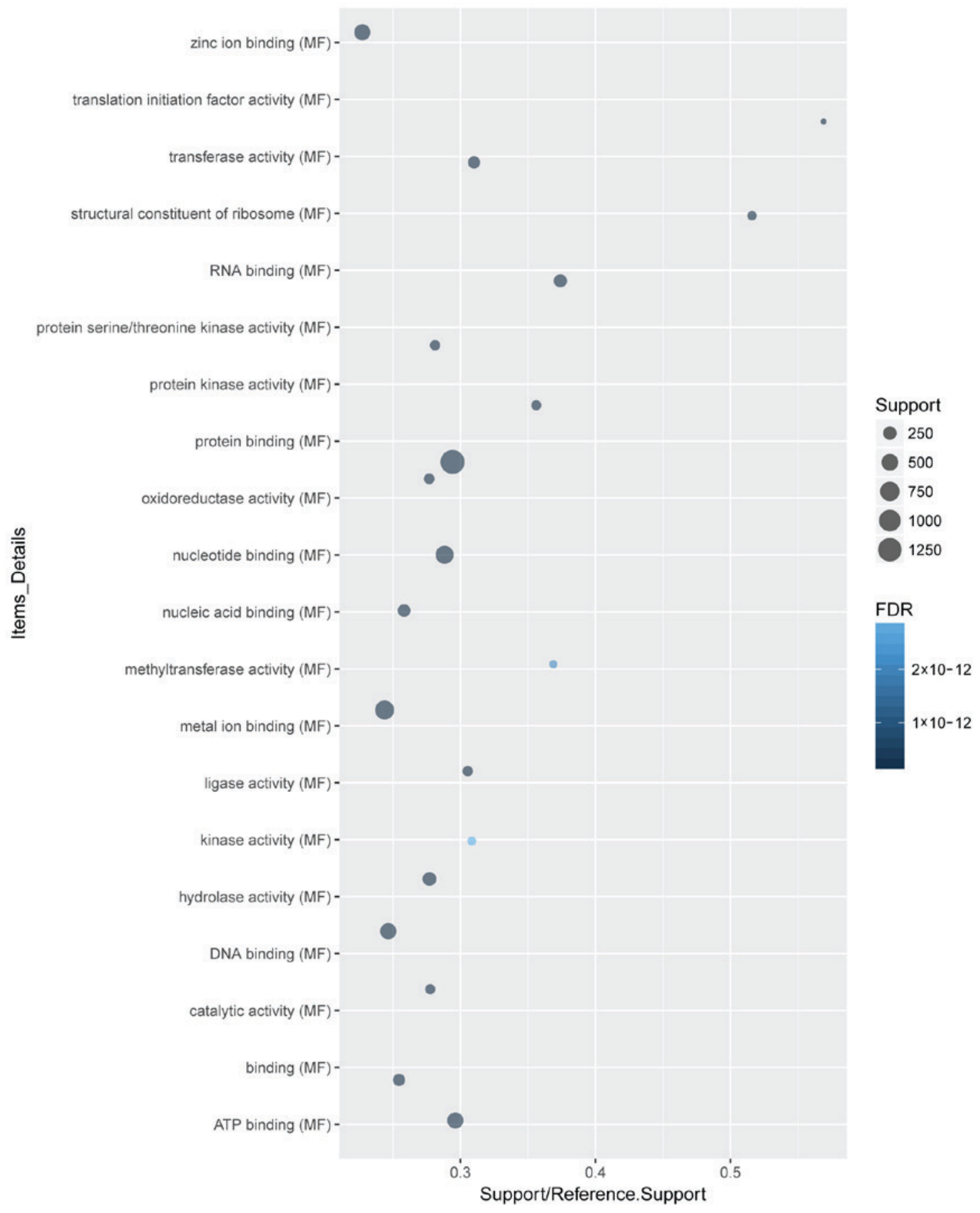

Figure 4. Top 20 significant MF terms that differentially expressed genes within GSE69528 and GSE46955 datasets were enriched in. MF, molecular function; GO, gene ontology; FDR, false discovery rate.

IRAK3 is an immune-associated enzyme. Notably, IRAK3 expression was elevated in blood monocytes from patients with sepsis, and expression levels in pediatric patients were directly associated with adverse clinical outcomes $(22,23)$. In addition, increased expression was also significantly increased following septic shock (24). Cazalis et al (24) identified four variants of the IRAK3 gene that were associated with acute lung injury development during severe sepsis. Targeting IRAK3 in sepsis may have an impact on the progression of this condition (25). The present study also identified a significant upregulation of IRAK3 expression in the blood samples of patients with sepsis compared with healthy controls. Furthermore,
IRAK3 was identified as a hub gene (degree, 18) in the PPI network for upregulated DEGs. Notably, it had a large value (AUC, 1) for sepsis diagnosis, confirming that IRAK3 may be critically involved in the development of sepsis and may be employed as a diagnostic marker and potential drug target in sepsis therapy.

ADM expression is induced by hypoxia, oxidative stress and proinflammatory cytokines. It exerts various effects, including the regulation of inflammation, infection and angiogenesis (26-29). ADM is also implicated in the vasodilation and hypotension that is associated with septic shock (30). Hirata et al (27) demonstrated that serum levels 


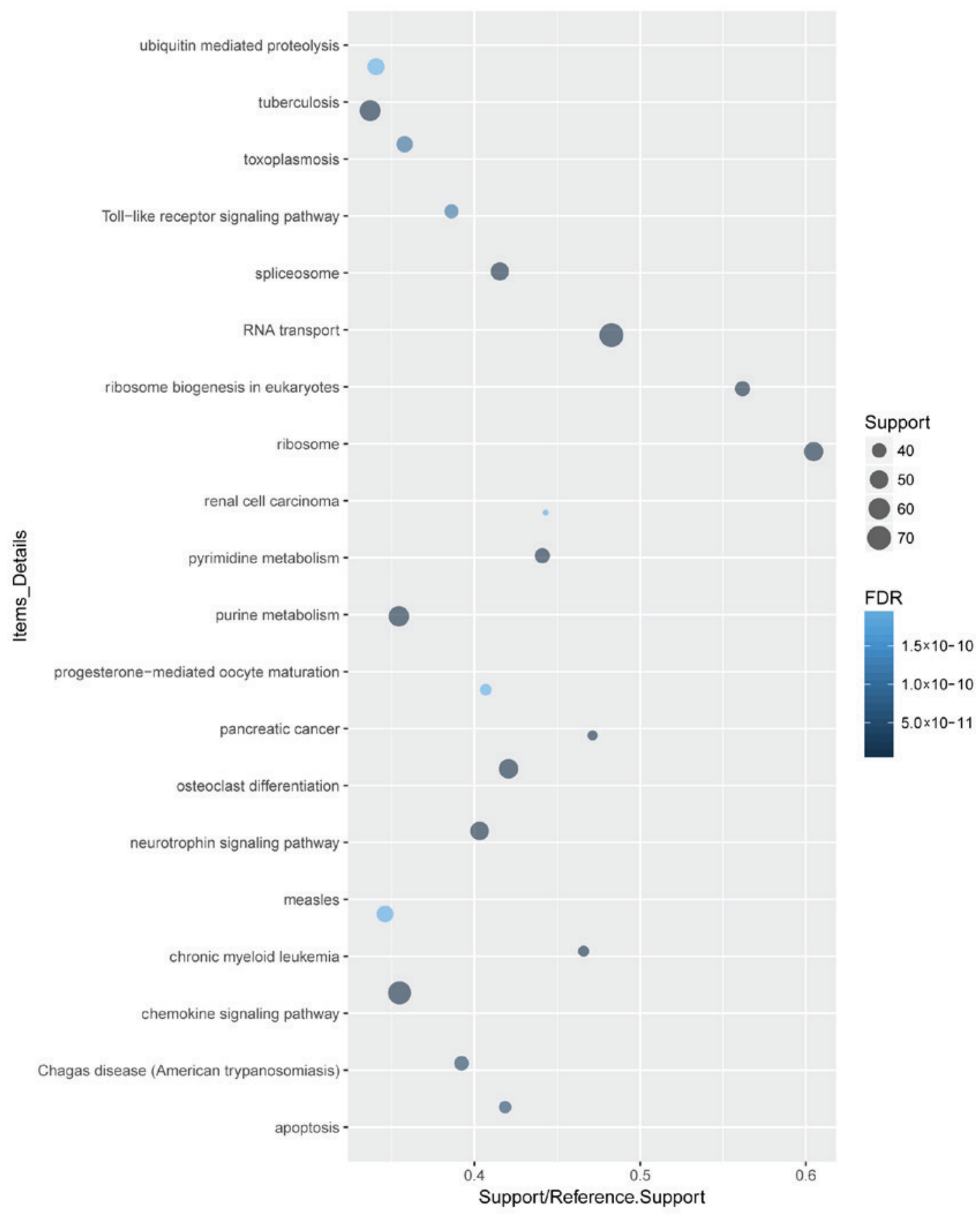

Figure 5. Top 20 significant KEGG signaling pathways that differentially expressed genes within GSE69528 and GSE46955 datasets were enriched in. KEGG, Kyoto Encyclopedia of Genes and Genomes; FDR, false discovery rate.

of ADM were increased in sepsis and the highest plasma concentrations of ADM were detected in patients with septic shock (26). It has been reported that ADM expression increased with increasing sepsis severity and was associated with increasingly adverse outcomes $(17,31)$. In the current study, the expression of ADM was demonstrated to be significantly increased in the blood sample data from patients with sepsis compared with healthy controls, which is consistent with a previous report (27). Furthermore, RT-qPCR analysis confirmed this increase in ADM expression, and diagnoses were also markedly associated with levels of ADM expression in patients with sepsis. The results of the present study highlighted the pivotal involvement of ADM in sepsis, which may aid the evaluation of sepsis diagnosis and prognosis.

ALOX5 expression is reported to be increased during inflammatory and immune responses $(32,33)$. It is typically expressed by leukocytes and is associated with the biosynthesis of leukotrienes. Various leukotriene mediators are thought to be pathogenic in inflammatory diseases such as sepsis (34). Monteiro et al (35) demonstrated that the products of ALOX5 induced lung injury during sepsis. Furthermore, ALOX5 expression in a mouse model exacerbated sepsis-induced multiple organ injury (36) and mice with an increased susceptibility to sepsis exhibited increased ALOX5 expression (37). In the present study, ALOX5 


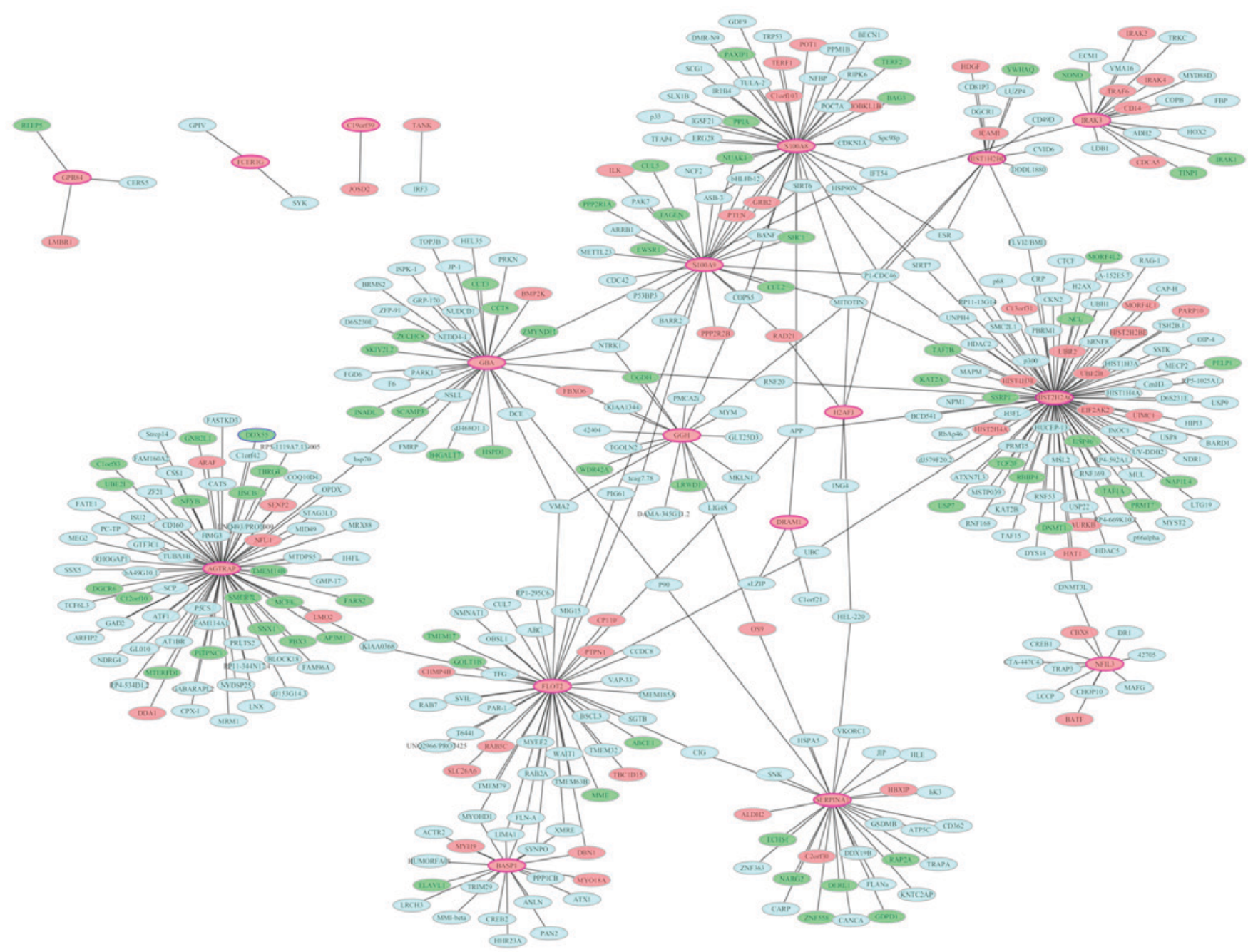

Figure 6. Protein-protein interaction networks of the top 20 upregulated DEGs within GSE69528 and GSE46955 datasets. All nodes indicate proteins encoded by DEGs and the pink borders represent proteins encoded by the top 20 upregulated DEGs. Green represents proteins encoded by the downregulated DEGs. Light blue represents proteins encoded by other genes that interact with these DEGs. The blue border represents proteins encoded by the top 20 downregulated DEGs. DEG, differentially expressed gene.

expression was significantly upregulated in blood samples from patients with sepsis compared with controls, and ALOX5 was determined to have a high diagnostic value, indicating that ALOX5 may have a crucial role in the inflammatory and immune processes of sepsis and has potential as a diagnostic marker.

It has been established that MMP9 is associated with inflammation and inhibits platelet aggregation (38-41). Previous studies demonstrated that the mRNA and protein expression levels of MMP9 in patients with sepsis were significantly higher compared with healthy individuals, and its expression levels have been investigated as prognostic biomarkers of sepsis (42-45). In the present study, RT-qPCR demonstrated that the expression of MMP9 was significantly upregulated in the blood samples from patients with sepsis compared with healthy controls, which was concordant with the bioinformatics analysis results. Furthermore, MMP9 was significantly associated with sepsis diagnosis, further demonstrating the function of MMP9 in the pathophysiology of sepsis.

S100A8 is an important inflammatory mediator (46), and a previous study has demonstrated that the expression levels of S100A8 is upregulated in sepsis (21). The peripheral blood level of S100A8 was elevated in patients with sepsis-associated encephalopathy (SAE) and may be associated with SAE severity (21). Furthermore, the presence of S100A8 in amniotic fluid was reported to be an important predictor of early-onset neonatal sepsis incidence (47-49). In the current study, S100A8 expression was significantly increased in blood samples of patients with sepsis compared with healthy controls and was also identified as a hub gene (degree, 43) in the PPI network of upregulated DEGs in sepsis. Notably, S100A8 had the largest AUC in sepsis diagnosis, indicating that S100A8 may have potential as a drug target and diagnostic marker in sepsis therapy.

ENTPD1 has been previously reported to reduce sepsis-associated mortality and improve the survival rate in microbial sepsis cases through the attenuation of systemic inflammation $(50,51)$. Additionally, numerous studies have demonstrated that ENTPD1 protected organs from hypoxic and non-infectious inflammation (52-55), which are implicated in the development of sepsis $(7,56)$. The present study identified that ENTPD1 expression was increased in sepsis compared with healthy controls in the GEO data; however, 


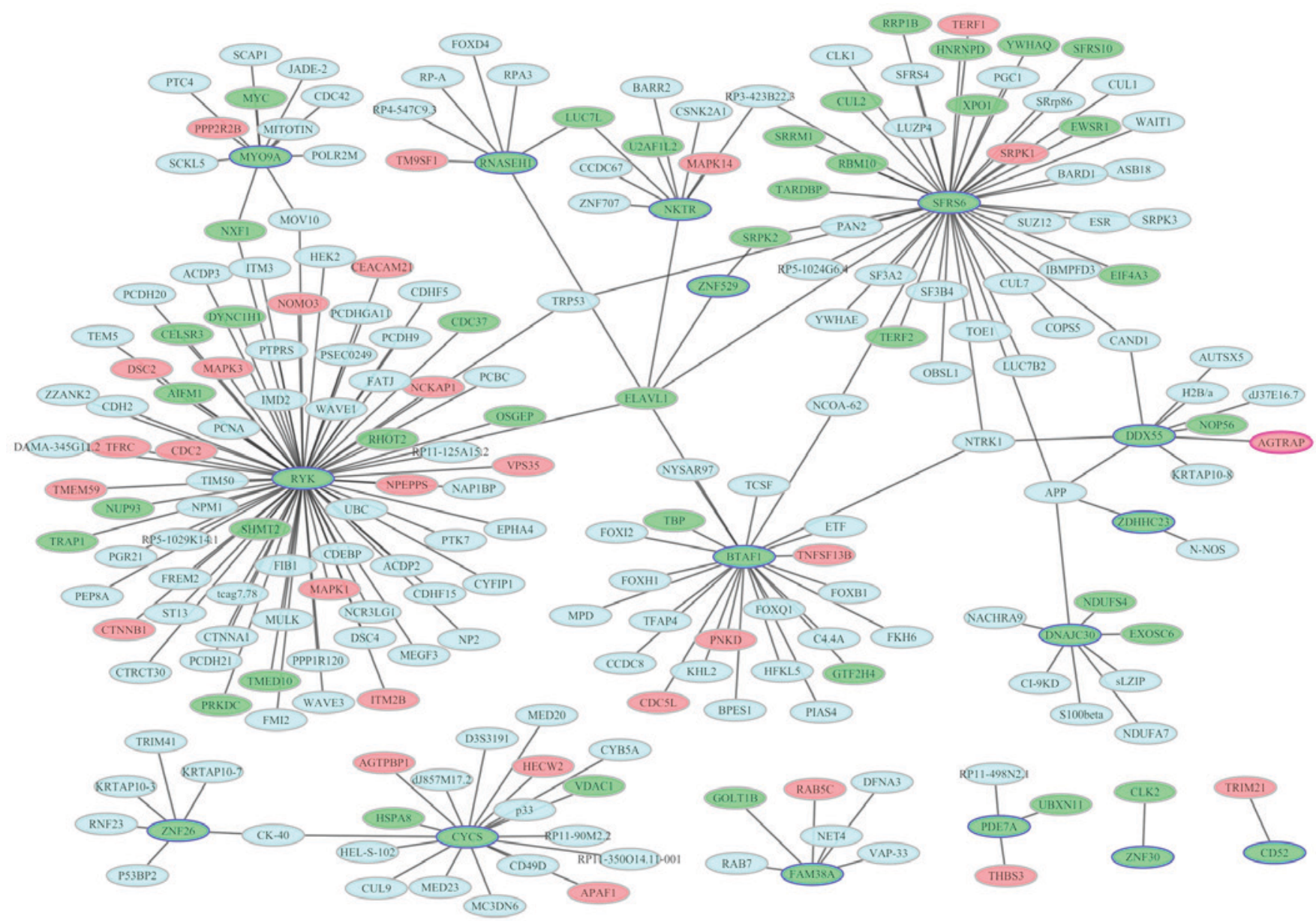

Figure 7. Protein-protein interaction networks of the top 20 downregulated DEGs within GSE69528 and GSE46955 datasets. All the nodes are proteins encoded by DEGs and the blue borders represent proteins encoded by the top 20 downregulated DEGs. Green represents proteins encoded by the down-regulated DEGs. Light blue. Light blue represent the proteins encoded by other genes that interact with these DEGs. Pink border represents proteins encoded by the top 20 downregulated DEGs. DEG, differentially expressed gene.

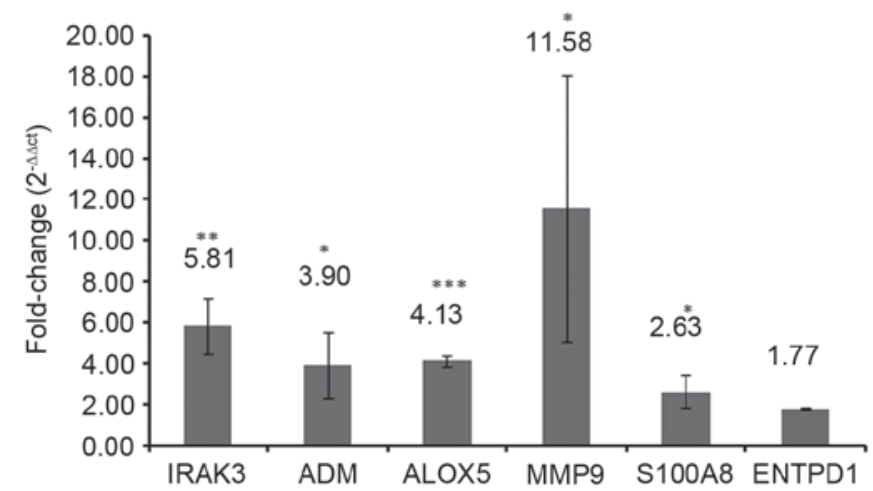

Figure 8. Expression levels of selected differentially expressed genes. Results are presented as fold change $\left(2^{-\Delta \Delta c q} ;\right.$ patient/control) values; $2^{-\Delta \Delta c q}>1$ indicates an upregulated gene and $2^{-\Delta \Delta c q}<1$ indicates a downregulated gene. IRAK3, interleukin 1 receptor-associated kinase 3; ADM, adrenomedullin; ALOX5 arachidonate 5-lipoxygenase; MMP9, matrix metallopeptidase 9; S100A8, S100 calcium-binding protein A8; ENTPD1, ectonucleoside triphosphate diphosphohydrolase 1 .

the increased expression was not significant in the RT-qPCR results. Therefore, further research is required to investigate the involvement of ENTPD1 in sepsis pathophysiology. Furthermore, for ENTPD1, a large diagnosis value for sepsis was calculated, which indicates that ENTPD1 may be employed as a diagnostic marker.

In addition to the above genes that were validated by RT-qPCR in the present study, AGTRAP and S100A9 were also identified to be associated with sepsis in the PPI network. AGTRAP has been reported to be implicated in septic shock caused by bacterial or viral infections (24). Nakada et al (57) also demonstrated that AGTRAP may be a potential pharmacogenetic biomarker in sepsis. S100A9 is a damage-associated molecule that has been reported to be a mediator of severe sepsis (58). In addition, S100A9 mRNA expression was increased in circulating cells in septic shock (59). Furthermore, S100A9 and S100A8 form a heterodimer that is implicated in multiple organ failure in septic shock (60). van Zoelen et al (61) demonstrated that the expression of S100A8/S100A9 was significantly elevated in the plasma of patients with severe sepsis. In the present study, the expression of AGTRAP and S100A9 was demonstrated to be upregulated in blood samples from patients with sepsis and to be implicated in sepsis development. AGTRAP was associated with a high diagnostic value with the highest AUC, indicating that AGTRAP may be a potential diagnostic marker for sepsis.

According to KEGG enrichment analysis, DEGs were demonstrated to be significantly enriched in the TLR 

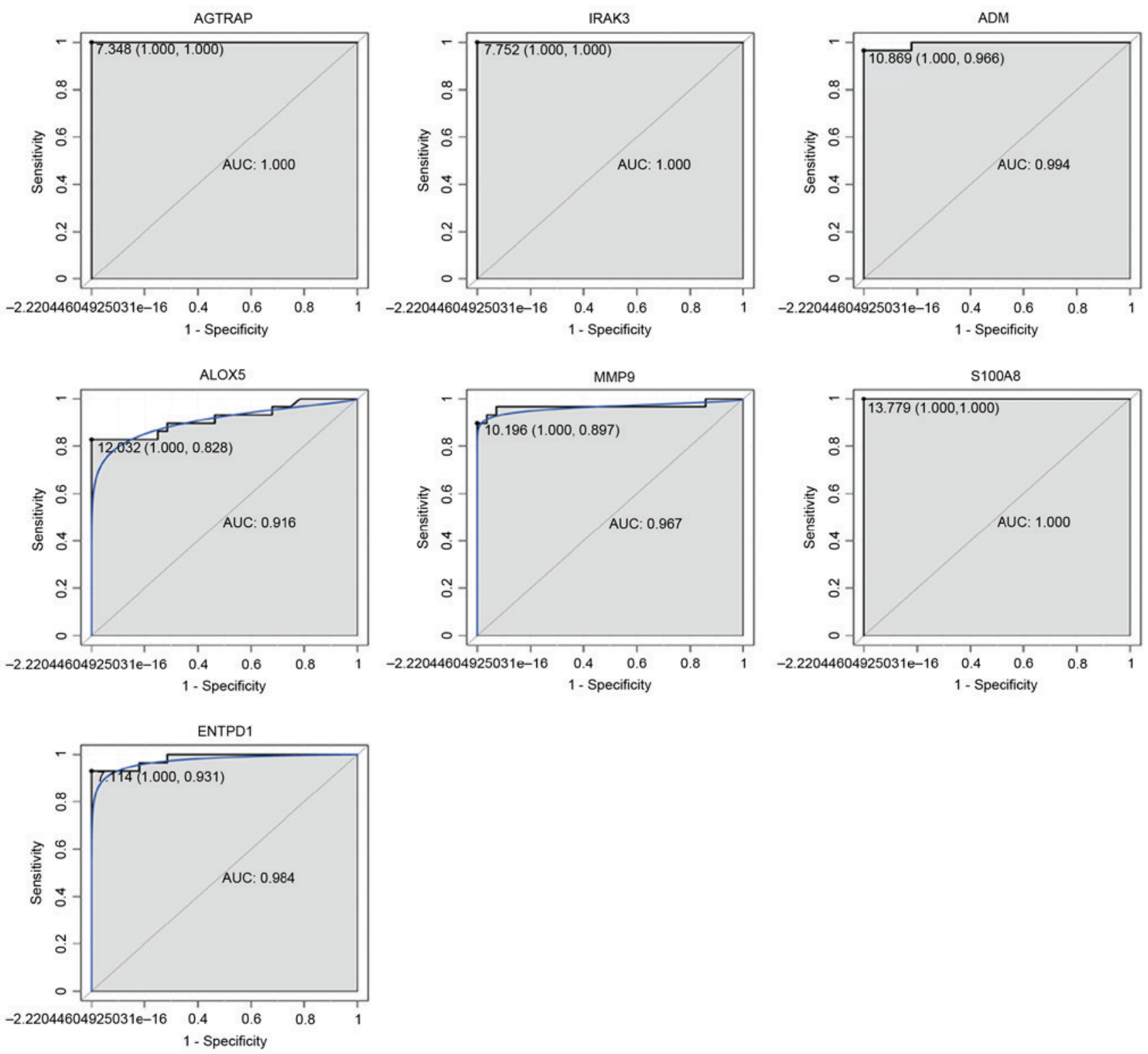

Figure 9. ROC curves of selected DEGs between patients with sepsis and healthy controls. The ROC curves were utilized to indicate the diagnostic ability of these selected DEGs with 1-Specificity (the proportion of false positive results; $x$-axis) and sensitivity (the proportion of true positive results; $y$-axis). ROC, receiver operating curve; DEG, differentially expressed gene; AGTRAP, angiotensin II receptor-associated protein; IRAK3, interleukin 1 receptor-associated kinase 3; ADM, adrenomedullin; ALOX5, arachidonate 5-lipoxygenase; MMP9, matrix metallopeptidase 9; S100A8, S100 calcium-binding protein A8; ENTPD1, ectonucleoside triphosphate diphosphohydrolase 1; AUC, area under the curve.

signaling pathway. In humans, at least ten different TLRs recognize specific microbial patterns to initiate inflammatory signaling pathways (62). TLRs are major contributors to sepsis development, particularly in the early stages where pro-inflammatory cytokines are produced (63). Savva and Roger (64) demonstrated that regulating the TLR/lymphocyte antigen 96-mediated inflammatory response may be a potential approach in the treatment and prevention of septic shock. Previous research has also demonstrated that TLR4 rs11536889 may be a marker of organ failure in sepsis (65).

Certain limitations are associated with the present study. The expression patterns in sepsis of two identified DEGs, ENTPD1 and AGTRAP, is unknown; therefore, future in vivo and in vitro experiments are required to investigate the expression and function of these genes in sepsis pathology. Additionally, studies with larger cohorts of patients with sepsis are required to confirm the diagnostic and therapeutic value of the identified genes. Larger numbers of blood samples are also required for further investigation to validate the RT-qPCR results obtained.

In conclusion, the results of the present study demonstrated that IRAK3, ADM, ALOX5, MMP9 and S100A8 expression was significantly upregulated in patients with sepsis compared with healthy controls, and they may therefore significantly contribute to the pathophysiology of sepsis. The identification of these genes may contribute to the development of early diagnostic tools, prognostic markers or therapeutic targets in sepsis.

\section{Acknowledgements}

The present study was funded by the Project Funds of Taizhou People's Hospital (grant no. ZL201716). 


\section{References}

1. Bao R, Shui X, Hou J, Li J, Deng X, Zhu X and Yang T: Adenosine and the adenosine A2A receptor agonist, CGS21680, upregulate CD39 and CD73 expression through E2F-1 and CREB in regulatory $\mathrm{T}$ cells isolated from septic mice. Int $\mathrm{J}$ Mol Med 38: 969-975, 2016

2. Jawad I, Lukšić I and Rafnsson SB: Assessing available information on the burden of sepsis: Global estimates of incidence, prevalence and mortality. J Glob Health 2: 010404, 2012.

3. Martin GS, Mannino DM and Moss M: The effect of age on the development and outcome of adult sepsis. Criti Care Med 34: 15-21, 2006.

4. Strehlow MC, Emond SD, Shapiro NI, Pelletier AJ and Camargo CA Jr: National study of emergency department visits for sepsis, 1992 to 2001. Ann Emerg Med 48: 326-331, 331.e1-e3, 2006.

5. Martin GS, Mannino DM, Eaton S and Moss M: The epidemiology of sepsis in the United States from 1979 through 2000. N Engl J Med 348: 1546-1554, 2003.

6. Murphy SL: Deaths: Final data for 1998. Natl Vital Stat Rep 48: $1-105,2000$

7. Hotchkiss RS and Karl IE: The pathophysiology and treatment of sepsis. N Engl J Med 348: 138-150, 2003.

8. Calvano SE, Xiao W, Richards DR, Felciano RM, Baker HV, Cho RJ, Chen RO, Brownstein BH, Cobb JP, Tschoeke SK, et al: A network-based analysis of systemic inflammation in humans. Nature 437: 1032-1037, 2005.

9. Wurfel MM, Gordon AC, Holden TD, Radella F, Strout J, Kajikawa O, Ruzinski JT, Rona G, Black RA, Stratton S, et al Toll-like receptor 1 polymorphisms affect innate immune responses and outcomes in sepsis. Am J Respir Crit Care Med 178: 710-720, 2008.

10. Angus DC, Linde-Zwirble WT, Lidicker J, Clermont G, Carcillo J and Pinsky MR: Epidemiology of severe sepsis in the United States: Analysis of incidence, outcome, and associated costs of care. Crit Care Med 29: 1303-1310, 2001

11. Pankla R, Buddhisa S, Berry M, Blankenship DM, Bancroft GJ, Banchereau J, Lertmemongkolchai $G$ and Chaussabel D: Genomic transcriptional profiling identifies a candidate blood biomarker signature for the diagnosis of septicemic melioidosis. Genome Biol 10: R127, 2009.

12. Wu JQ, Sassé TR, Wolkenstein G, Conceicao V, Saksena MM, Soedjono M, Perera SS, Wang B, Dwyer DE and Saksena NK Transcriptome analysis of primary monocytes shows global down-regulation of genetic networks in HIV viremic patients versus long-term non-progressors. Virology 435: 308-319, 2013

13. Marot G, Foulley JL, Mayer CD and Jaffrézic F: Moderated effect size and $\mathrm{P}$-value combinations for microarray meta-analyses. Bioinformatics 25: 2692-2699, 2009.

14. Reiner-Benaim A: FDR control by the $\mathrm{BH}$ procedure for two-sided correlated tests with implications to gene expression data analysis. Biom J 49: 107-126, 2007.

15. Benjamini $\mathrm{Y}$ and Hochberg Y: Controlling the false discovery rate-a practical and powerful approach to multiple testing. J Royal Stat Soc 57: 289-300, 1995.

16. Schmittgen TD and Livak KJ: Analyzing real-time PCR data by the comparative C(T) method. Nat Protoc 3: 1101-1108, 2008.

17. Pino-Yanes M, Ma SF, Sun X, Tejera P, Corrales A, Blanco J, Pérez-Méndez L, Espinosa E, Muriel A, Blanch L, et al: Interleukin-1 receptor-associated kinase 3 gene associates with susceptibility to acute lung injury. Am J Respir Cell Mol Biol 45: 740-745, 2011.

18. Kesik V, Atas E, Gülcan Kurt Y, Aydın FN, Babacan O, Gülgün $\mathrm{M}$ and Korkmazer N: Adrenomedullin predicts high risk and culture positivity in children with solid tumors suffering from neutropenic fever. J Infect Chemother 22: 617-621, 2016.

19. Pavanelli WR, Gutierrez FR, Mariano FS, Prado CM, Ferreira BR, Teixeira MM, Canetti C, Rossi MA, Cunha FQ and Silva JS: 5-lipoxygenase is a key determinant of acute myocardial inflammation and mortality during Trypanosoma cruzi infection. Microbes Infect 12: 587-597, 2010

20. Shogan BD, Belogortseva N, Luong PM, Zaborin A, Lax S, Bethel C, Ward M, Muldoon JP, Singer M, An G, et al: Collagen degradation and MMP9 activation by Enterococcus faecalis contribute to intestinal anastomotic leak. Sci Transl Med 7: 286ra268, 2015
21. Zhang LN, Wang XH, Wu L, Huang L, Zhao CG, Peng QY and Ai YH: Diagnostic and predictive levels of calcium-binding protein A8 and tumor necrosis factor receptor-associated factor 6 in sepsis-associated encephalopathy: A prospective observational study. Chin Med J (Engl) 129: 1674-1681, 2016.

22. Hall MW, Gavrilin MA, Knatz NL, Duncan MD, Fernandez SA and Wewers MD: Monocyte mRNA phenotype and adverse outcomes from pediatric multiple organ dysfunction syndrome. Pediatr Res 62: 597-603, 2007.

23. Escoll P, del Fresno C, García L, Vallés G, Lendínez MJ, Arnalich F and López-Collazo E: Rapid up-regulation of IRAK-M expression following a second endotoxin challenge in human monocytes and in monocytes isolated from septic patients. Biochem Biophys Res Commun 311: 465-472, 2003.

24. Cazalis MA, Lepape A, Venet F, Frager F, Mougin B, Vallin H, Paye M, Pachot A and Monneret G: Early and dynamic changes in gene expression in septic shock patients: A genome-wide approach. Intensive Care Med Exp 2: 20, 2014.

25. Deng JC, Cheng G, Newstead MW, Zeng X, Kobayashi K, Flavell RA and Standiford TJ: Sepsis-induced suppression of lung innate immunity is mediated by IRAK-M. The J Clin Invest 116: 2532-2542, 2006.

26. Ueda S, Nishio K, Minamino N, Kubo A, Akai Y, Kangawa K, Matsuo H, Fujimura Y, Yoshioka A, Masui K, et al: Increased plasma levels of adrenomedullin in patients with systemic inflammatory response syndrome. Am J Respir Crit Care Med 160: 132-136, 1999.

27. Hirata Y, Mitaka C, Sato K, Nagura T, Tsunoda Y, Amaha K and Marumo F: Increased circulating adrenomedullin, a novel vasodilatory peptide, in sepsis. J Clin Endocrinol Metabol 81: 1449-1453, 1996

28. Sugo S, Minamino N, Shoji H, Kangawa K, Kitamura K, Eto $\mathrm{T}$ and Matsuo $\mathrm{H}$ : Interleukin-1, tumor necrosis factor and lipopolysaccharide additively stimulate production of adrenomedullin in vascular smooth muscle cells. Biochem Biophys Res Commun 207: 25-32, 1995.

29. Musson DS, McLachlan JL, Sloan AJ, Smith AJ and Cooper PR: Adrenomedullin is expressed during rodent dental tissue development and promotes cell growth and mineralization. Biol Cell 102: 145-157, 2010.

30. So S, Hattori Y, Kasai K, Shimoda S and Gross SS: Up-regulation of rat adrenomedullin gene expression by endotoxin: Relation to nitric oxide synthesis. Life sciences 58: Pl309-P1315, 1996.

31. Simon TP, Martin L, Doemming S, Humbs A, Bruells C, Kopp R, Hartmann O, Struck J, Bergmann A, Marx G and Schuerholz T: Plasma adrenomedullin in critically ill patients with sepsis after major surgery: A pilot study. J Crit Care 38: 68-72, 2017.

32. Talwar S, Munson PJ, Barb J, Fiuza C, Cintron AP, Logun C, Tropea M, Khan S, Reda D, Shelhamer JH, et al: Gene expression profiles of peripheral blood leukocytes after endotoxin challenge in humans. Physiol Genomics 25: 203-215, 2006.

33. Prabhakar U, Conway TM, Murdock P, Mooney JL, Clark S, Hedge P, Bond BC, Jazwinska EC, Barnes MR, Tobin F, et al: Correlation of protein and gene expression profiles of inflammatory proteins after endotoxin challenge in human subjects. DNA Cell Biol 24: 410-431, 2005.

34. Peters-Golden M and Henderson WR Jr: Leukotrienes. N Engl J Med 357: 1841-1854, 2007.

35. Monteiro AP, Soledade E, Pinheiro CS, Dellatorre-Teixeira L, Oliveira GP, Oliveira MG, Peters-Golden M, Rocco PR, Benjamim CF and Canetti C: Pivotal role of the 5-lipoxygenase pathway in lung injury after experimental sepsis. Am J Respir Cell Mol Biol 50: 87-95, 2014.

36. Collin M, Rossi A, Cuzzocrea S, Patel NS, Di Paola R, Hadley J, Collino M, Sautebin L and Thiemermann C: Reduction of the multiple organ injury and dysfunction caused by endotoxemia in 5-lipoxygenase knockout mice and by the 5-lipoxygenase inhibitor zileuton. J Leukoc Biol 76: 961-970, 2004.

37. Das UN: Combination of aspirin with essential fatty acids is superior to aspirin alone to prevent or ameliorate sepsis or ARDS. Lipids Health Dis 15: 206, 2016.

38. Stamenkovic I: Extracellular matrix remodelling: The role of matrix metalloproteinases. J Pathol 200: 448-464, 2003.

39. Chakrabarti S and Patel KD: Regulation of matrix metalloproteinase-9 release from IL-8-stimulated human neutrophils. J Leukoc Biol 78: 279-288, 2005.

40. Sheu JR, Fong TH, Liu CM, Shen MY, Chen TL, Chang Y, Lu MS and Hsiao G: Expression of matrix metalloproteinase-9 in human platelets: Regulation of platelet activation in in vitro and in vivo studies. Br J Pharmacol 143: 193-201, 2004. 
41. Lee YM, Lee JJ, Shen MY, Hsiao G and Sheu JR: Inhibitory mechanisms of activated matrix metalloproteinase- 9 on platelet activation. Eur J Pharmacol 537: 52-58, 2006.

42. Jin LY, Li CF, Zhu GF, Wu CT, Wang J and Yan SF: Effect of siRNA against NF- $\kappa B$ on sepsisinduced acute lung injury in a mouse model. Mol Med Rep 10: 631-637, 2014

43. Cizmeci MN, Kara S, Kanburoglu MK, Simavli S, Duvan CI and Tatli MM: Detection of cord blood hepcidin levels as a biomarker for early-onset neonatal sepsis. Med Hypotheses 82: 310-312, 2014.

44. Hoffmann U, Bertsch T, Dvortsak E, Liebetrau C, Lang S, Liebe V, Huhle $G$, Borggrefe $M$ and Brueckmann $M$ : Matrix-metalloproteinases and their inhibitors are elevated in severe sepsis: Prognostic value of TIMP-1 in severe sepsis. Scand J Infect Dis 38: 867-872, 2006.

45. Edgar JD, Gabriel V, Gallimore JR, McMillan SA and Grant J: A prospective study of the sensitivity, specificity and diagnostic performance of soluble intercellular adhesion molecule 1, highly sensitive C-reactive protein, soluble E-selectin and serum amyloid $\mathrm{A}$ in the diagnosis of neonatal infection. BMC Pediatr 10: 22, 2010

46. Gebhardt C, Nemeth J, Angel P and Hess J: S100A8 and S100A9 in inflammation and cancer. Biochem Pharmacol 72: 1622-1631, 2006.

47. Buhimschi IA and Buhimschi CS: The role of proteomics in the diagnosis of chorioamnionitis and early-onset neonatal sepsis. Clin Perinatol 37: 355-374, 2010.

48. Buhimschi CS, Buhimschi IA, Abdel-Razeq S, Rosenberg VA, Thung SF, Zhao G, Wang E and Bhandari V: Proteomic biomarkers of intra-amniotic inflammation: Relationship with funisitis and early-onset sepsis in the premature neonate. Pediatr Res 61: 318-324, 2007.

49. Buhimschi CS, Bhandari V, Dulay AT, Nayeri UA, Abdel-Razeq SS, Pettker CM, Thung S, Zhao G, Han YW, Bizzarro M and Buhimschi IA: Proteomics mapping of cord blood identifies haptoglobin 'switch-on' pattern as biomarker of early-onset neonatal sepsis in preterm newborns. PLoS One 6 : e26111, 2011

50. Csóka B, Németh ZH, Törő G, Koscsó B, Kókai E, Robson SC, Enjyoji K, Rolandelli RH, Erdélyi K, Pacher P and Haskó G: CD39 improves survival in microbial sepsis by attenuating systemic inflammation. FASEB J 29: 25-36, 2015.

51. Haskó G, Csóka B, Koscsó B, Chandra R, Pacher P Thompson LF, Deitch EA, Spolarics Z, Virág L, Gergely P, et al: Ecto-5'-nucleotidase (CD73) decreases mortality and organ injury in sepsis. J Immunol 187: 4256-4267, 2011.

52. Eltzschig HK, Thompson LF, Karhausen J, Cotta RJ, Ibla JC, Robson SC and Colgan SP: Endogenous adenosine produced during hypoxia attenuates neutrophil accumulation: Coordination by extracellular nucleotide metabolism. Blood 104: 3986-3992, 2004.

53. Köhler D, Eckle T, Faigle M, Grenz A, Mittelbronn M, Laucher S, Hart ML, Robson SC, Müller CE and Eltzschig HK: CD39/ectonucleoside triphosphate diphosphohydrolase 1 provides myocardial protection during cardiac ischemia/reperfusion injury. Circulation 116: 1784-1794, 2007.
54. Grenz A, Zhang H, Hermes M, Eckle T, Klingel K, Huang DY, Müller CE, Robson SC, Osswald $\mathrm{H}$ and Eltzschig HK: Contribution of E-NTPDase1 (CD39) to renal protection from ischemia-reperfusion injury. FASEB J 21: 2863-2873, 2007.

55. Hart ML, Gorzolla IC, Schittenhelm J, Robson SC and Eltzschig HK: SP1-dependent induction of CD39 facilitates hepatic ischemic preconditioning. J Immunol 184: 4017-4024, 2010.

56. Riedemann NC, Guo RF and Ward PA: The enigma of sepsis. J Clin Invest 112: 460-467, 2003.

57. Nakada TA, Russell JA, Boyd JH, McLaughlin L, Nakada E, Thair SA, Hirasawa H, Oda S and Walley KR: Association of angiotensin II type 1 receptor-associated protein gene polymorphism with increased mortality in septic shock. Crit Care Med 39: 1641-1648, 2011.

58. Mares CA, Ojeda SS, Morris EG, Li Q and Teale JM: Initial delay in the immune response to Francisella tularensis is followed by hypercytokinemia characteristic of severe sepsis and correlating with upregulation and release of damage-associated molecular patterns. Infect Immun 76: 3001-3010, 2008.

59. Fontaine M, Pachot A, Larue A, Mougin B, Landelle C, Venet F, Allombert C, Cazalis MA, Monneret G and Lepape A: Delayed increase of S100A9 messenger RNA predicts hospital-acquired infection after septic shock. Crit Care Med 39: 2684-2690, 2011.

60. Feuerstein GZ: Cardiac RAGE in sepsis: Call TOLL free for anti-RAGE. Circ Res 102: 1153-1154, 2008.

61. van Zoelen MA, Vogl T, Foell D, Van Veen SQ, van Till JW, Florquin S, Tanck MW, Wittebole X, Laterre PF, Boermeester MA, et al: Expression and role of myeloid-related protein-14 in clinical and experimental sepsis. Am J Respir Crit Care Med 180: 1098-1106, 2009.

62. West AP, Koblansky AA and Ghosh S: Recognition and signaling by toll-like receptors. Ann Rev Cell Dev Biol 22: 409-437, 2006.

63. Buchholz BM and Bauer AJ: Membrane TLR signaling mechanisms in the gastrointestinal tract during sepsis. Neurogastroenterol Motil 22: 232-245, 2010.

64. Savva A and Roger T: Targeting toll-like receptors: Promising therapeutic strategies for the management of sepsis-associated pathology and infectious diseases. Front Immunol 4: 387, 2013.

65. Mansur A, von Gruben L, Popov AF, Steinau M, Bergmann I, Ross D, Ghadimi M, Beissbarth T, Bauer M and Hinz J: The regulatory toll-like receptor 4 genetic polymorphism rs11536889 is associated with renal, coagulation and hepatic organ failure in sepsis patients. J Transl Med 12: 177, 2014.

This work is licensed under a Creative Commons Attribution-NonCommercial-NoDerivatives 4.0 International (CC BY-NC-ND 4.0) License. 